\title{
BMJ Open Scientific publications in respiratory journals from Chinese authors in various parts of North Asia: a 10-year survey of literature
}

\author{
Bo Ye, ${ }^{1}$ Ting-Ting Du, ${ }^{1}$ Ting Xie, ${ }^{2}$ Jun-Tao Ji, ${ }^{1}$ Zhao-Hong Zheng, ${ }^{1}$ Zhuan Liao, ${ }^{1}$ \\ Liang-Hao Hu, ${ }^{1}$ Zhao-Shen $\mathrm{Li}^{1}$
}

To cite: Ye B, Du T-T, Xie T, et al. Scientific publications in respiratory journals from Chinese authors in various parts of North Asia: a 10-year survey of literature. BMJ Open 2014;4:e004201. doi:10.1136/ bmjopen-2013-004201

- Prepublication history for this paper is available online. To view these files please visit the journal online (http://dx.doi.org/10.1136/ bmjopen-2013-004201).

BY, T-TD and TX have contributed equally.

Received 9 October 2013 Revised 3 February 2014 Accepted 10 February 2014

CrossMark

\footnotetext{
${ }^{1}$ Department of Internal Medicine, Changhai Hospital, the Second Military Medical University, Shanghai, China ${ }^{2}$ Department of Internal Medicine, Zhongda Hospital the Southeast University, Nanjing, China
}

Correspondence to Dr. Zhao-Shen Li, zhaoshen-li@hotmail.coml; Dr. Liang-Hao Hu, lianghao-hu@hotmail.com

\section{ABSTRACT}

Objectives: Respiratory disease remains one of the leading causes of morbidity and mortality in China. However, little is known about the research status of respirology in three major regions of China-Mainland (ML), Hong Kong (HK) and Taiwan (TW). A 10-year survey of literature was conducted to compare the three regions' outputs in the research of respirology.

Design: A bibliometric study.

Setting: China.

Participants and outcome measures: A literature search in PubMed database, updated as of September 2012, led to the identification of the related articles from 2000 to 2009. The number of total articles, randomised controlled trials, case reports, metaanalysis, impact factors (IF), citations and articles published in top general medicine journals was collected for quantity and quality comparisons.

Results: 2208 articles were collected, 814 from ML, 909 from TW and 485 from HK. The total number of articles from the three regions has increased significantly from 2000 to 2009 . The number of articles published per year from ML has exceeded that from HK in 2005 and TW in 2008. The accumulated IF of articles from TW (3192.417) was much higher than that from ML (2409.956) and HK (1898.312). HK got the highest average IF of respirology articles and the majority of articles were published in top general medicine journals.

Conclusions: The total number of published articles from the three major regions of China has increased notably from 2000 to 2009 . The annual number of publications by ML researchers exceeded those from TW and HK. However, the quality of articles from TW and $\mathrm{HK}$ is better than that from ML.

\section{INTRODUCTION}

Pulmonary diseases are a matter of concern for all the countries. Lung disease accounts for $15 \%$ of disability-adjusted life years ${ }^{1}$ and may be the first cause of mortality in the world. $^{2} 3$

\section{Strengths and limitations of this study}

A few journals covered resources beyond respiratory even selected from the respiratory system of Science Citation Index Expanded (SCIE). Besides, some related journals not shown in SCIE were not collected. Some respiratory medicine research articles were published in general journals, rather than in the specialized ones. Searching by the author's address (China, HK or TW) led to another problem that the articles which addressed other cities or provinces were not included. Actually, our previous design had taken Macau into consideration, but later we excluded it for its small amount and low correlation.

In China, under the influence of the dramatic economic evolution and urbanisation progress, the levels and patterns of outdoor and indoor air pollutants have greatly changed. Hundreds of cities suffer the substandard air quality according to the WHO guidelines. ${ }^{4}$ Besides, with a population of 1.3 billion, China has become a major contributor to the worldwide respiratory disease burden. ${ }^{5}{ }^{6}$ In terms of the fatality rate, respiratory disease comes the first in rural areas and the fourth in urban areas in China. However, little is known about the research status of respirology in the three major regions of China-Mainland (ML), Hong Kong (HK) and Taiwan (TW). ${ }^{7} \mathrm{We}$, therefore, conducted a 10-year survey of literature and compared these three regions outputs in the research of respirology.

\section{MATERIALS AND METHODS}

A total of 46 journals related to respirology were selected from the 'respiratory system' category of Science Citation Index Expanded (SCIE) subject categories by the Institute for Scientific Information (ISI). ${ }^{8}$ The category covers resources dealing with the diagnosis 
and treatment of respiratory disease and focuses on prevention, pharmacology, surgery, transplantation and research. Annals of Thoracic Medicine, COPD and Portuguese Journal of Pulmonology (Revista Portuguesa de Pneumologia) were not indexed by PubMed, so they were excluded. A computerised literature search was conducted in the PubMed database on 10 September 2012 (http://www.ncbi.nlm.nih.gov/pubmed/).

Articles from ML, TW and HK from January 2000 to December 2009 in these journals were elicited, respectively. We used the ISSN to perform searches in PubMed. The search terms used were (1040-0605 OR 1073-449X OR 1044-1549 OR 1817-1737 OR 0003-4975 OR 03002896 OR 1198-2241 OR 0012-3692 OR 1752-6981 OR 0272-5231 OR 1541-2555 OR 1070-5287 OR 1010-7940 OR 0903-1936 OR 0190-2148 OR 0147-9563 OR 10273719 OR 1806-3713 OR 1941-2711 OR 0277-0903 OR 1053-0770 OR 1569-1993 OR 1053-2498 OR 0022-5223 OR 1556-0864 OR 0341-2040 OR 0169-5002 OR 1828695X OR 1526-0542 OR 8755-6863 OR 1094-5539 OR 0025-7931 OR 0020-1324 OR 0954-6111 OR 1569-9048 OR 1465-9921 OR 1323-7799 OR 0873-2159 OR 07618417 OR 0761-8425 OR 1124-0490 OR 1069-3424 OR 1520-9512 OR 0171-6425 OR 0040-6376 OR 1472-9792 AND TAIWAN [AD] AND 2000[DP] AND 'Hong Kong $[\mathrm{AD}]$ ', 'Taiwan [AD]', and 'China [AD] NOT Hong Kong [AD] NOT Taiwan [AD]'. Articles that showed the first author's affiliation (AD) with these three regions were considered as research outputs from the regions. Articles in the fields of randomised controlled trials (RCT), review, meta-analysis and case reports were generated, respectively, according to the publication types by PubMed.

To compare the quality of the research articles, three methods were used: (1) the accumulated impact factors (IF) and the average IF were generated according to Journal Citation Reports (JCR) 2010 established by the ISI (http://isiknowledge.com); (2) citation reports of articles showing an affiliation with a Chinese institution were conducted; (3) articles published in top general medicine journals (The New England Journal of Medicine (NEJM), Journal of the American Medical Association (JAMA), The Lancet and British Medical Journal (BMJ)) were also generated. Articles related to respirology were first extracted independently by two reviewers (T-TD and L-HH), and any disagreement between the reviewers was resolved by viewing the titles, abstracts and full text if necessary. The number of articles published by each region in the top 10 high-impact respiratory journals was also compared. We determined the 10 most popular respiratory journals containing articles from the three regions of China according to the number of such articles published by each journal.

\section{Statistical analysis}

Statistical analyses were performed using STATA V.11.0. ${ }^{9}$ The non-parametric test for trend and time series analysis was performed to established change of the total

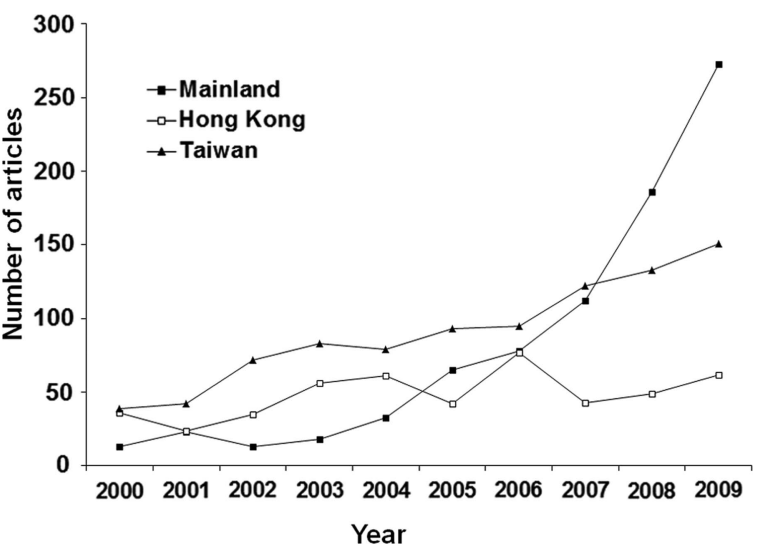

Figure 1 The trend of the number of articles from Mainland, Taiwan and Hong Kong during the past 10 years.

numbers over the period of time. Kruskal-Wallis test was used for detecting the difference among the three regions, and rank-sum test between two if necessary. The test for significance was two-tailed and the value of $\mathrm{p}<0.05$ was considered significant.

\section{RESULTS}

\section{Total number of articles}

A total number of 83787 articles were published in the selected 46 journals within the period 2000-2009 worldwide. There were 2208 articles $(2208 / 83787,2.64 \%)$ from ML (814/2208, 36.9\%), TW (909/2208 41.1\%) and HK $(485 / 2208,22.0 \%)$. The numbers increased significantly from 2001 to 2010 in the three regions (from 13 to $273, \mathrm{p}=0.004$ for trend, 39 to $151, \mathrm{p}=0.003,36$ to $62, \mathrm{p}=0.047$, respectively, figure 1 ). From 2005 onwards, the number of articles from ML has exceeded that from HK and in 2008 ML exceeded TW. The share of articles was on the rise in ML $(p=0.001)$ and TW $(p=0.040)$, but not in HK ( $\mathrm{p}=0.813$, figure 2$)$.

\section{Randomised controlled trial, review, meta-analysis} and case report

In respect of randomised controlled trial (figure 3), it shows no difference among the three regions (ML vs TW

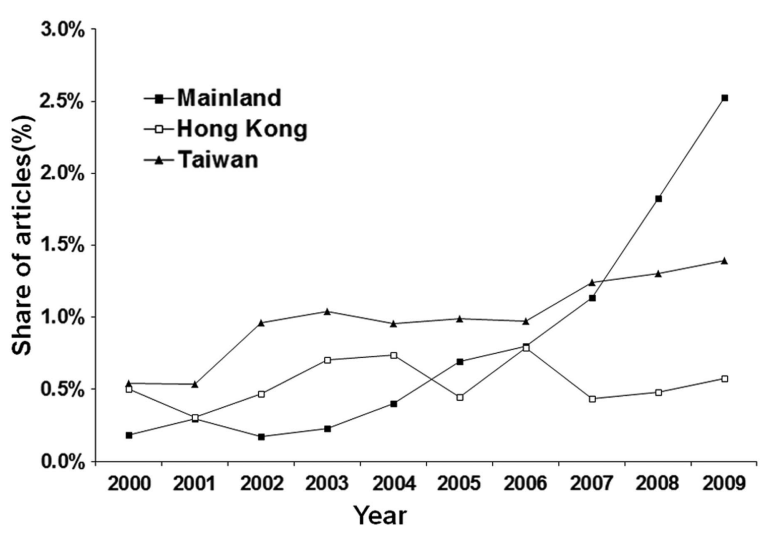

Figure 2 The share of articles from Mainland, Taiwan and Hong Kong during the past 10 years. 


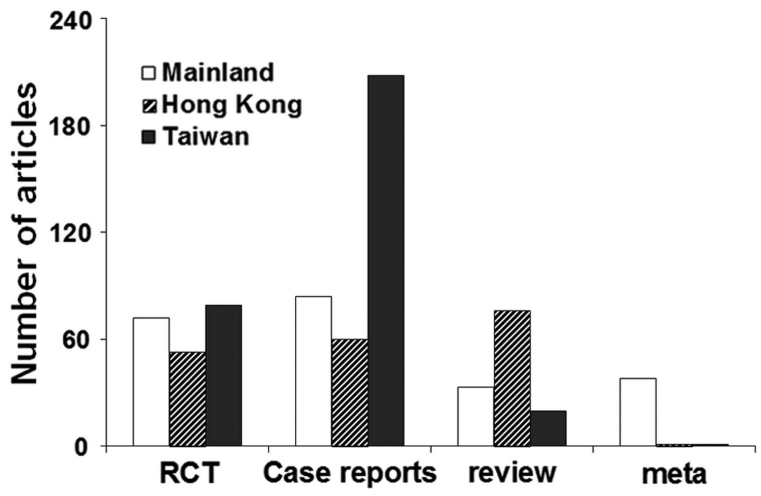

Figure 3 Number of articles on randomised controlled trial, case report, review and meta-analysis from Mainland, Taiwan and Hong Kong.

vs $\mathrm{HK}, \mathrm{p}=0.3662$ ). In respect of review, ML and TW (ML vs TW $\mathrm{p}=0.7591$ ), although less than HK, showed no significant difference (ML vs HK $\mathrm{p}=0.0331$; TW vs HK $\mathrm{p}=0.0038$ ). Besides, we found a number of meta-analysis from ML since 2006, but few from TW and HK. However, as per this case report, ML and HK (ML vs HK p=0.6215) were equal to each other, while less than TW (TW vs HK $\mathrm{P}=0.0006$; TW vs ML $\mathrm{P}=0.0064$ ).

\section{Impact factors}

According to the JCR, 43 journals in the 'respiratory system' had IF in 2010 (http://isiknowledge.com). Three journals in our study had no IF. Excluding them, the accumulated IF of articles from TW (3192.417) was much higher than that from ML (2409.956) and HK (1898.312, $\mathrm{p}=0.038)$. However, HK took the highest average IF of 3.914, followed by TW of 3.512 and ML of $2.961(\mathrm{p}=0.003$, table 1$)$.

\section{Citation reports of articles published in the} respiratory journals

The ISI has not set up a function for finding a citation report of articles by limiting the department of the corresponding author, so in this citation reports, the articles included were affiliated with a Chinese institution, more than the previous search results in PubMed. ${ }^{10}$ According to our analysis, TW had the highest total citations of 6320 (1064 articles), followed by HK with 5232 total citations in 700 articles and ML with 5232 citations in 1122 articles. These differences among the three regions were not significant ( $\mathrm{p}=0.772$; figure 4 ).

\section{High-impact respiratory journals and top general medicine journals}

Of 1014 articles from these three regions, which were published in the 10 top-ranking respiratory journals, $40.33 \%(409 / 1014)$ were in American Journal of Respiratory and Critical Care Medicine, Thorax, Chest and European Respiratory Journal. TW published 456 articles in the high IF respiratory journals, while ML and HK had 336 and 222 articles, respectively, in the top 10 journals (table 2). A total number of 271 articles (ML 81, TW 71 and HK 119) were published in the four top general medicine journals (NEJM, Lancet, JAMA and BMJ). Fifty-five articles in the field of respirology were selected by the two reviewers. HK owned the most articles, with a total of 31 (original articles 23 including 1 RCT and 1 guideline, review 1 , case reports 2, others 5; NEJM 6, JAMA 1, Lancet 16 and BMJ 8), ML had 16 articles (original articles 12 including 3 RCTs, case report 1 , others 3; NEJM 3, JAMA 1, Lancet 10 and BMJ 2) and TW had 8 articles (original articles 3, case reports 5; NEJM 5, JAMA 1 and Lancet 2).

\section{Popular respiratory journals}

The most popular journals in the three regions are shown in table 3. Chest ranked the first in HK and TW, while Annals of Thoracic Surgery ranked the first in ML. Annals of Thoracic Surgery, European Journal of CardioThoracic Surgery, Respirology, The International Journal of Tuberculosis and Lung Disease and Chest are all ranked top 10.

Table 1 The accumulated and average impact factor of articles from Mainland (ML), Taiwan (TW) and Hong Kong (HK)

\begin{tabular}{|c|c|c|c|c|c|c|}
\hline \multirow[b]{2}{*}{ Year } & \multicolumn{3}{|c|}{ Accumulated impact factor } & \multicolumn{3}{|c|}{ Average impact factor } \\
\hline & $\overline{M L}$ & HK & TW & $\overline{\mathrm{ML}}$ & HK & TW \\
\hline 2000 & 54895 & 167831 & 171309 & 4.223 & 4.662 & 4.393 \\
\hline 2001 & 66619 & 78862 & 149393 & 2.896 & 3.286 & 3.557 \\
\hline 2002 & 38238 & 133937 & 248554 & 2.941 & 3.827 & 3.452 \\
\hline 2003 & 50552 & 194997 & 328783 & 2.808 & 3.482 & 3.961 \\
\hline 2004 & 109898 & 244190 & 331810 & 3.330 & 4.003 & 4.200 \\
\hline 2005 & 218684 & 162832 & 355665 & 3.364 & 3.877 & 3.824 \\
\hline 2006 & 242293 & 308506 & 318215 & 3.106 & 4.007 & 3.350 \\
\hline 2007 & 292890 & 193632 & 379165 & 2.615 & 4.503 & 3.108 \\
\hline 2008 & 533500 & 172982 & 463555 & 2.868 & 3.530 & 3.485 \\
\hline 2009 & 802387 & 240543 & 445968 & 2.939 & 3.880 & 2.953 \\
\hline TOTAL & 2409956 & 1898312 & 3192417 & 2.961 & 3.914 & 3.512 \\
\hline
\end{tabular}




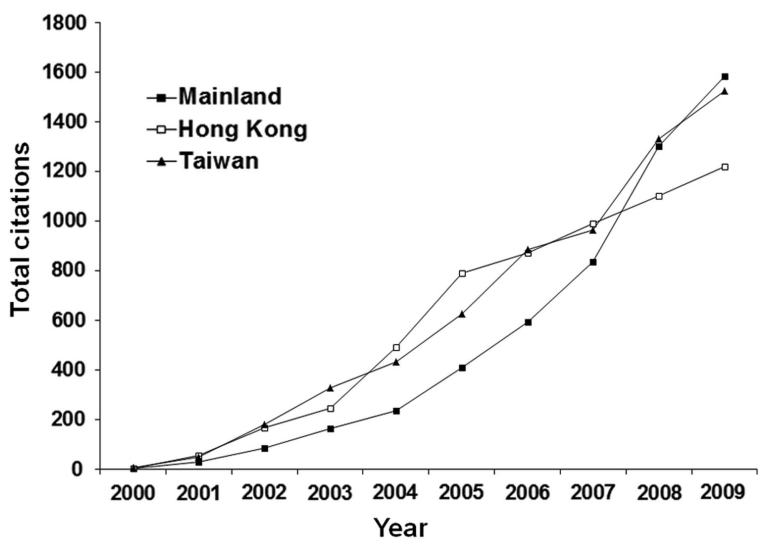

Figure 4 Total citations of articles from Mainland, Taiwan and Hong Kong published on respiratory journals from 2000 to 2009 .

\section{DISCUSSION}

To the best of our knowledge, this is the first report that showed clearly the contributions of Chinese authors in major regions of China-ML, $\mathrm{HK}$ and $\mathrm{TW}-$ to the worldwide research in the field of respirology. Our study results showed that the number of published articles from ML China had increased significantly in the past 10 years, and surpassed HK in 2005 and TW in 2008. However, when impact factors (IF), citation reports and articles published in top general medicine journals were taken into quality comparisons, the gap among the three regions appeared wide. HK had the highest average IF of 3914, followed by TW of 3512 and ML of 2961, mainly because the readers worldwide preferred English, which gave HK an advantage over the other two regions. The number of articles from China in the 'respiratory system' category ranked 11 worldwide in total $(2.64 \%$ for share), although a substantial number of high-quality articles were in Chinese. In addition, the situation that the articles increased in the field of respirology was also found in other fields, such as Cardiology, Cardiovasology and Gastroenterology. ${ }^{11} 12$
The three regions equal to each other in terms of randomised controlled trial for the past decades. However, ML has gradually come to the fore since 2007. In 2008, ML released more reports than TW and $\mathrm{HK}$, indicating its obvious superiority of large population and relatively low research cost. ${ }^{13}$

However, the published essays, in fact, are just a small part of randomised controlled trials achievements. The reasons are as follows: the records were dispersed not only in the Chinese Clinical Trial Register (ChiCTR), but also in other WHO International Clinical Trials Registry Platform (ICTPR) primary registries or International Committee of Medical Journal Editors (ICMJE) approved registries. ${ }^{14}$ Besides, part of the results were released in Chinese or in the journals not indexed by PubMed. Moreover, the randomised controlled trials in China showed a lower to an average publication rate. ${ }^{15-17}$

China, in recent years, is prospering rapidly economically to become the second largest economy in the world after the USA. However, air pollution, especially hazes, followed this prosperity. The Global Burden of Disease Study 2010 found that particulate matter with an aerodynamic diameter of less than $2.5 \mu \mathrm{m}$ (PM 2.5) has become the fourth biggest threat to the health of the Chinese people. ${ }^{18}$ Although smoking has fallen in China, the prevalence of lung cancer has increased, most probably due to air pollution. ${ }^{19}$ China, currently, faces an arduous task of addressing the challenges of environmental pollution. ${ }^{20}$ The pace of life is increasing with technological advancements, and this leads to hypertension and decreased exercise in people's daily life. All of these risk factors obviously will increase the morbidity of respiratory diseases. There is a price that developing countries must pay for modernisation. However, let the price of the Chinese pay not exceed the benefits from modernisation. Therefore, government, scientific association and doctors pay more attention to the study about respiratory diseases. ${ }^{21} 22$

The data in our study mainly came from two sources: the PubMed search system and the JCR. The PubMed

Table 2 Articles published on the 10 most influential journals from Mainland (ML), Taiwan (TW) and Hong Kong (HK)

\begin{tabular}{|c|c|c|c|c|c|c|c|c|c|}
\hline Rank & Journal & 2009 IF & ML & $(\%)$ & TW & (\%) & HK & (\%) & Total \\
\hline 1 & Am J Respir Crit Care & 10.689 & 8 & 15 & 20 & 38 & 24 & 46 & 52 \\
\hline 2 & Thorax & 7.041 & 9 & 15 & 18 & 30 & 33 & 55 & 60 \\
\hline 3 & Chest & 6.360 & 28 & 13 & 127 & 58 & 65 & 30 & 220 \\
\hline 4 & Eur Respir J & 5.527 & 17 & 22 & 24 & 31 & 36 & 47 & 77 \\
\hline 5 & J Thorac Oncol & 4.547 & 19 & 59 & 9 & 28 & 4 & 13 & 32 \\
\hline 6 & Am J Respir Cell Mol & 4.319 & 12 & 29 & 21 & 51 & 8 & 20 & 41 \\
\hline 7 & Am J Physiol Lung C & 4.043 & 7 & 29 & 16 & 67 & 1 & 4.2 & 24 \\
\hline 8 & Ann Thorac Surg & 3.644 & 115 & 44 & 109 & 42 & 37 & 14 & 261 \\
\hline 9 & J Heart Lung Transpl & 3.541 & 9 & 32 & 15 & 54 & 4 & 14 & 28 \\
\hline 10 & Lung Cancer & 3.140 & 112 & 51 & 97 & 44 & 10 & 5 & 219 \\
\hline
\end{tabular}

Am J Respir Crit Care Med, American Journal of Respiratory and Critical Care Medicine; Eur Respir J, European Respiratory Journal; $J$ Thorac Oncol, Journal of Thoracic Oncology, Am J Respir Cell Mol, American Journal of Respiratory Cell and Molecular Biology; Am J Physiol Lung C, American Journal of Physiology — Lung Cellular and Molecular Physiology; Ann Thorac Surg, Annals of Thoracic Surgery, $J$ Heart Lung Transpl, The Journal of Heart and Lung Transplantation. 
Table 3 The 10 most popular respiratory journals in Mainland (ML), Taiwan (TW) and Hong Kong (HK)

\begin{tabular}{|c|c|c|c|c|c|c|}
\hline Rank & $\begin{array}{l}M L \\
(n=814)\end{array}$ & $\mathbf{N}$ & TW $(n=909)$ & $\mathbf{N}$ & $\begin{array}{l}\text { HK } \\
(n=485)\end{array}$ & $\mathbf{N}$ \\
\hline 1 & ATS & 115 & Chest & 127 & Chest & 65 \\
\hline 2 & $L C$ & 112 & ATS & 109 & Respirology & 62 \\
\hline 3 & EJCT & 68 & $L C$ & 97 & IJTLD & 42 \\
\hline 4 & JTCS & 68 & JTCS & 93 & ATS & 37 \\
\hline 5 & Respirology & 57 & EJCT & 59 & $E R J$ & 36 \\
\hline 6 & Respiration & 41 & Respirology & 42 & Thorax & 33 \\
\hline 7 & IJTLD & 34 & IJTLD & 39 & $R M$ & 30 \\
\hline 8 & Chest & 28 & $P P$ & 36 & $P P$ & 25 \\
\hline 9 & $R R$ & 23 & TCS & 36 & AJRCC & 24 \\
\hline 10 & PPT & 22 & $J A$ & 29 & EJCT & 19 \\
\hline
\end{tabular}

ATS, Annals of Thoracic Surgery. IF=3644; LC, Lung Cancer. IF=3140; EJCT, European Journal of Cardio-Thoracic Surgery: IF=2397; JTCS, Journal of Thoracic and Cardiovascular Surgery: IF=3063; Respirology. IF=1853; Respiration: IF=1935; IJTLD, The International Journal of Tuberculosis and Lung Disease: IF=2548; Chest. IF=636; RR, Respiratory Research: IF=3127; PPT, Pulmonary Pharmacology and Therapeutics: IF=2024; PP, Pediatric Pulmonology. IF=1816; TCS, Thoracic and Cardiovascular Surgeon: IF=0745; JA, Journal of Asthma: $\mathrm{IF}=1372$; ERJ, European Respiratory Journal: IF=5527; Thorax. IF=7041; RM, Respiratory Medicine: IF=2331; AJRCC, American Journal of Respiratory and Critical Care Medicine: IF=10 689.

search system is a comprehensive database run by the National Center for Biotechnology Information (NCBI) at the National Library of Medicine in Bethesda, Maryland, containing articles from high-quality medical journals. The JCR, published by the Institute for Scientific Information in 1975, represents the most comprehensive citation index to the scientific literature and covers more than 7000 journals at 2010. Although IF is not the optimal parameter for determining the quality of articles, it is, at present, the best available parameter for judging the quality of studies.

Our study has its limitations, however. A few journals covered resources beyond respiratory even selected from the respiratory system of SCIE. Besides, some related journals not shown in SCIE were not collected. Some respiratory medicine research articles are published in general journals, rather than in the specialised ones. ${ }^{23}$

Searching by the author's address (China, HK or TW) led to another problem that the articles which addressed other cities or provinces were not included. Actually, our previous design had taken Macau into consideration, but later we excluded it for its small amount and low correlation (1 in Heart Lung, 2007 and 1 in Respirology, 2009).

In conclusion, the number of articles published from ML, TW and HK has increased significantly during the past 10 years. There has also been an appreciable increase in the total number of articles from the three major regions of China from 2000 to 2009. The number of articles published per year from ML has exceeded that from TW and HK. However, the quality of articles from TW and HK is better than that from ML.

Contributors L-HH conceived and designed the study. BY was involved in data search and selection of data, analysed the data and wrote the manuscript. T-TD was involved in data search, selection of data and analysed the data. TX was involved in writing the manuscript. J-TJ was involved in data search and analysed the data. Z-HZ and ZL were involved in data search and selection of data. Z-SL analysed the data and contributed analysis tools.
Funding This research received no specific grant from any funding agency in the public, commercial or not-for-profit sectors.

Competing interests None.

Provenance and peer review Not commissioned; externally peer reviewed.

Data sharing statement No additional unpublished data are available.

Open Access This is an Open Access article distributed in accordance with the Creative Commons Attribution Non Commercial (CC BY-NC 3.0) license, which permits others to distribute, remix, adapt, build upon this work noncommercially, and license their derivative works on different terms, provided the original work is properly cited and the use is non-commercial. See: http:// creativecommons.org/licenses/by-nc/3.0/

\section{REFERENCES}

1. Guntupalli KK, Gutterman D, Raoof S, et al. 2010: the year of the lung. Chest 2010;138:1287-8.

2. Sandoval-Gutierrez JL, Reyes ES, Bautista EB. Pulmonary diseases: first cause of mortality in the world. Chest 2011;139:1550.

3. Mathers CD, Boerma T, Ma Fat D. Global and regional causes of death. Br Med Bull 2009;92:7-32.

4. Chinese Academy of Engineering, Chinese Academy of Sciences, National Academy of Engineering, National Research Council. Proceedings of a Symposium Introduction of urbanization, energy and air pollution in China: the Challenges Ahead. The National Academies Press. Washington, DC, 2003:1-3.

5. Fang $X$, Wang $X$, Bai $C$. COPD in China: the burden and importance of proper management. Chest 2011;139:920-9.

6. Sun YC. Taking ACTion for better control of asthma. Chin Med J (Engl) 2007;120:1035-6.

7. Soteriades ES, Falagas ME. A bibliometric analysis in the fields of preventive medicine, occupational and environmental medicine, epidemiology, and public health. BMC Public Health 2006;15:301.

8. Journal search: Science citation index expanded subject categories. Cited 10 Sep 2012. http://scientific.thomsonreuters.com/cgibin/jrnlst/ jlresults.cgi

9. STATA statistical software, version 11.0, Stata Corp, College Station, TX, USA

10. Li G, Hu LH, Liao Z, et al. Scientific publications in pharmacology and pharmacy journals from Chinese authors in various parts of North Asia: 10-year survey of literature. J Int Med Res 2010;38:750-9.

11. Goh KL, Farrell GC. Publications from China: the sleeping giant awakens. J Gastroenterol Hepatol 2008;23:341-3.

12. Hu LH, Liao Z, Gao R, et al. Scientific publications in cardiology and cardiovasology journals from Chinese authors in various parts of North Asia: 10-year survey of literature. Int J Cardiol 2010;140:304-8.

13. Mervis J. China's unique environment favors large intervention trials. Science 1995;270:1149-51. 
14. Liu XM, Li YP, Yu XT, et al. Assessment of registration quality of trials sponsored by China. J Evid Based Med 2009;2:8-18.

15. Song FJ, Parekh-Bhurke S, Hooper L, et al. Extent of publication bias in different categories of research cohorts: a meta-analysis of empirical studies. BMC Med Res Methodol 2009;9:79.

16. Xuemei L, Youping L, Shangqi S, et al. Ethical review reporting of Chinese trials records in WHO primary registries. J Med Ethics 2011;37:144-8.

17. Michalopoulos A, Falagas ME. A bibliometric analysis of global research production in respiratory medicine. Chest 2005;128:3993-8.

18. Cancer death report in China: the third national retrospective sampling death survey People's Medical Publishing House, Beijing. National Office for Cancer Prevention and Control National Center for Cancer Registries Bureau of Disease Prevention and Control Ministry of Health, 2008.

19. Xu P. Haze, air pollution, and health in China. Lancet 2013;382:2067.

20. Chen Z, Wang JN, Ma GX, et al. China tackles the health effects of air pollution. Lancet 2013;382:1959-60.

21. Yao X, Sun $Y$. The sounds of small airways: emerging role in pathogenesis and clinical expression of asthma. Chin Med J (Engl) 2014;127:173-9.

22. Sun YC. Advances in respiratory medicine in the mainland of China: a historical perspective. Chin Med J (Engl) 2010;123:6-17.

23. Partridge MR, Lewison G. Respiratory research output. Chest 2006;130:1283. 